\title{
Brentuximab Vedotin
}

\author{
National Cancer Institute
}

\section{Source}

National Cancer Institute. Brentuximab Vedotin. NCI Thesaurus. Code C66944.

An antibody-drug conjug ate (ADC) directed ag ainst the tumor necrosis factor (TNF) receptor CD30 with potential antineoplastic activity. Brentuximab vedotin is generated by conjug ating the chimeric anti-CD30 monoclonal antibody SGN-30 to the cytotoxic agent monomethyl auristatin E (MMAE) via a valine-citrulline peptide linker. Upon administration and internalization by CD30-positive tumor cells, brentuximab vedotin undergoes enzymatic cleavage, releasing MMAE into the cytosol; MMAE binds to tubulin and inhibits tubulin polymerization, which may result in G2/M phase arrest and tumor cell apoptosis. Transiently activated during lymphocyte activation, CD30 (tumor necrosis factor receptor superfamily, member 8;T NFRSF8) may be constitutively expressed in hematologic malignancies including Hodgkin lymphoma and some T-cell non-Hodgkin lymphomas. The linkage system in brentuximab vedotin is highly stable in plasma, resulting in cytotoxic specificity for CD30-positive cells. 